\title{
Component Optimization for Catalyst Layers in Proton Exchange Membrane Fuel Cells
}

\author{
Jiaheng Peng, ${ }^{a}$ Wencong Zhang, ${ }^{a}$ Xuewei Zhang, ${ }^{*, b}$ Peng Tao, ${ }^{a}$ Chengyi Song, ${ }^{a}$ Wen Shang, ${ }^{a}$ Tao Deng, ${ }^{a, c, d}$ \\ and Jianbo $\mathrm{Wu}^{*, a, c, d}$ \\ a State Key Laboratory of Metal Matrix Composites, School of Materials Science and Engineering, \\ Shanghai Jiao Tong University, 800 Dongchuan Rd, Shanghai 200240, China \\ ${ }^{b}$ Weichai Power Co., Ltd., 197 Fushoudong Rd, Weifang, Shandong 261000, China \\ ${ }^{c}$ Center of Hydrogen Science, Shanghai Jiao Tong University, Shanghai 200240, China \\ ${ }^{d}$ Materials Genome Initiative Center, Shanghai Jiao Tong University, Shanghai 200240, China
}

Email: zhangxuew@weichai.com (X. Z.); jianbowu@sjtu.edu.cn (J. W.)

\begin{abstract}
Proton exchange membrane fuel cells (PEMFCs) have been recognized as a promising energy conversion solution. The biggest challenges for the commercialization of PEMFCs are cost and durability. Although many efforts have been made on catalysts, the performance and durability of the membrane electrode assemblies (MEAs) still cannot fully meet the targets established by the U.S. Department of Energy. Optimizing the catalyst layer to maximize the utilization of catalysts is a quite practical issue. In this paper, we reviewed the most recent advances on the component optimization for catalyst layers, including support materials, ionomers, and solvents. Based on the reaction mechanism on the three-phase boundary, enhancing the intrinsic properties of each component and optimizing their interactions can attribute a lot to the performance and durability of PEMFCs.
\end{abstract}

Keywords proton exchange membrane fuel cells, catalyst layers, support materials, ionomers, solvents

\section{Introduction}

Proton exchange membrane fuel cells (PEMFCs), due to their high power conversion efficiency, low operating temperature, and zero-emission, have been recognized as a promising energy conversion solution, especially for vehicle applications. ${ }^{[1]}$ Pt-based catalysts have long been regarded as the best candidate for PEMFCs. However, widespread commercialization of PEMFCs is limited by the cost and scarcity of platinum in the catalysts. Furthermore, the performance and durability of PEMFCs require further improvement. Although much progress has been made on the intrinsic design of catalysts with reduced platinum loading, high activity, and high durability, there are still significant barriers required to be overcome before the commercial application of PEMFCs. ${ }^{[2,3]}$ As reported in recent literatures, the electrochemical surface area (ECSA) and mass activity (MA) of the advanced catalysts measured by single-cell tests are only a fraction of those measured by rotating disk electrode (RDE), which suggests catalysts are underutilized in membrane electrode assemblies (MEAs). ${ }^{[4]}$ To narrow the gap between RDE and MEAs, the fundamentals and influence factors of MEAs, especially the catalyst layers, need to be further studied.

In recent years, several emerging methods of fabricating catalyst layers such as electrodeposition, ${ }^{[5]}$ sputtering, ${ }^{[6]}$ and dual ion-beam assisted deposition ${ }^{[7]}$ have been reported to produce efficient electrodes. However, the traditional ink-based method, which fabricates a thin catalyst layer on the proton exchange membrane (PEM) or the gas diffusion layer (GDL) with the ink consisting of the catalyst, the support material, the ionomer, and the solvent, is still the most widely used, owing to its convenience and high efficiency.

The catalyst layer of PEMFCs prepared by the ink-based method consists of the support material network, the ionomer

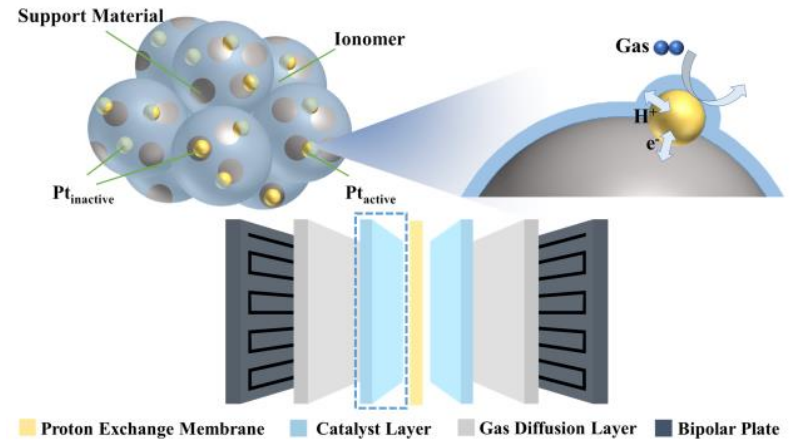

Figure 1 Scheme of the "three-phase boundary" on the catalyst layer of PEMFCs.

network, and the pore network, as shown in Figure 1. The electrode reactions in MEAs take place on the "three-phase boundary", where the active catalyst particles simultaneously contact with the support material network, the ionomer network, and the pore network. During the process of the electrode reactions, the catalysts absorb or release electrons after capturing the reactants, which are transported via the pore network. Subsequently, the electrons are transferred through the support material network, while the protons are transferred through the ionomer network. The capability of absorbing or releasing electrons is determined by the intrinsic catalyst activity. Besides, mass transfer, electron transfer, and proton transfer can also determine the performance of PEMFCs, which can be directly affected by the components and the interaction in the catalyst layers. The catalyst particles that are completely or not covered by the ionomers would lose activities because of poor mass transfer or proton transfer, respectively. Therefore, 
the component optimization for catalyst layers is significant to enhance the performance and long-term durability of PEMFCs.

Herein, we will review the most recent advances on the component optimization of the catalyst ink from the view of the whole catalyst layer instead of just separate parts to achieve a more efficient transfer of electrons, protons, and reactants. Along this line, the strategies to modify the catalyst layer will be analyzed and summarized from three aspects, including support materials, ionomers, and solvents.

\section{Support Materials}

During the past years, many efforts have focused on the development of efficient catalysts, including $\mathrm{Pt} / \mathrm{C}$, Pt-based alloy/dealloy, core-shell, ${ }^{[8]}$ nonprecious metal catalysts, ${ }^{[9]}$ shape-controlled nanocrystals, ${ }^{[10]}$ and nanoframes. ${ }^{[11]}$ The cathode catalyst layer of nonprecious metal catalysts is much thicker than that of Pt-based catalysts, which results in the transport loss in the catalyst layer. Therefore, Pt-based catalysts are still regarded as the most promising candidate for the commercialization of PEMFCs (Figure 2). ${ }^{[12]}$ However, the performance gap, especially the durability of catalysts obtained by RDE and MEAs, is an outstanding challenge that needs to be further explored.

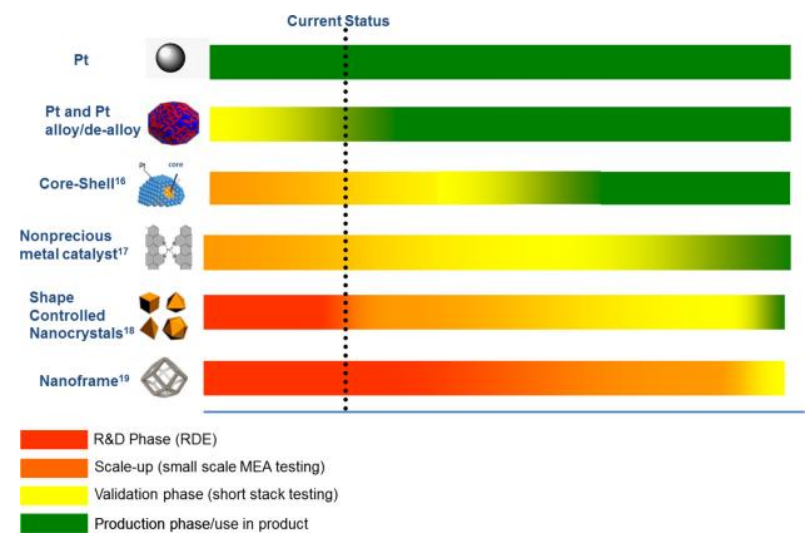

Figure 2 Current status and evolution for $\mathrm{Pt}$, Pt-based alloy/dealloy, core-shell, nonprecious metal, shape-controlled, and nanoframe electrocatalysts. ${ }^{[12]}$ Reprinted with permission from Ref. [12]. Copyright 2017 American Chemical Society.

The performance degradation in the MEA test is primarily related to the limited stability of electrocatalysts and electrode structures (Figure 3). Carbon corrosion and metal dissolution/sintering are the main mechanisms leading to the instability of electrocatalysts. Cheap carbon is selected as the typical support material because it can provide a large surface area and high electrical conductivity. Previous studies have been focused on commercial Pt-based catalysts supported on carbon black (CB), but the corrosion and oxidation of the carbon black under the harsh working conditions lead to the degradation of supported metal catalysts, which shortens the lifetime of PEMFCs. The corrosion of carbon support also leads to the thinner catalyst layers and changes its hydrophobicity, which changes the orientation of ionomer and hinders efficient water management. The damage of the ionic pathway caused by the corrosion of carbon also weakens the contact of reaction species. The stable metal particles anchored uniformly on the support play a crucial role in maintaining efficiency. Therefore, the stability study of carbon support has attracted increasing attention.

To achieve a high performing fuel cell, the support materials for catalyst tested in MEA are expected to provide high surface area, high electrochemical and chemical stability, and high

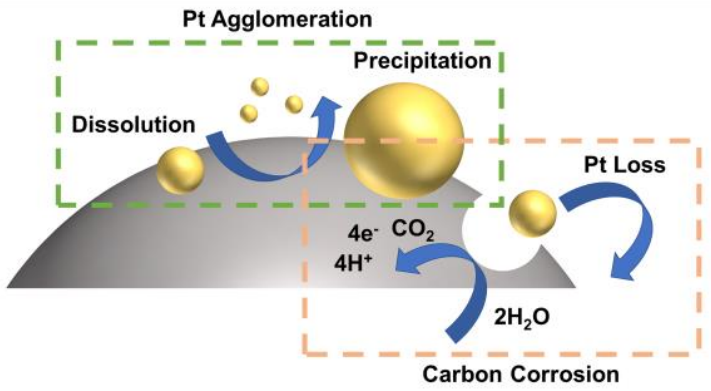

Figure 3 Schematic of the degradation of catalyst, including Pt agglomeration and carbon corrosion.

conductivity to ensure the homogeneous distribution of catalysts and abundant electron transfer. For these reasons, carbon nanotubes, carbon nanofibers, ${ }^{[13,14]}$ graphene, ${ }^{[15]}$ mesoporous carbon, ${ }^{[16]}$ carbon aerogels, ${ }^{[17]}$ and their corresponding derivates are studied widely in recent reports. ${ }^{[18]}$ The well-designed carbon structures are found to improve the performance not only by altering the intrinsic properties of support materials but also by enhancing the metal-support interaction.

Based on the structure design, surface modification can also be applied to further optimize the intrinsic activity of carbons. Lu et al. ${ }^{[19]}$ reported a novel multi-wall carbon nanotubes (MWCNTs) structure by introducing oxygencontaining functional groups (ketonic $\mathrm{C}=\mathrm{O}$ ) on their outer wall. The electron structures of neighboring carbon atoms can be adjusted by the function group $\mathrm{C}=\mathrm{O}$ existing on the MWCNTs to facilitate the adsorption of reaction intermediates during the oxygen evolution reaction (OER). This surface modification can be broadened to rational design of oxygen reduction reaction (ORR) catalysts.

Heteroatom doping into the carbon materials is another strategy to induce the redistribution of charge and adjust the chemisorption of $O$ species. Gong et al. ${ }^{[20]}$ pioneered that the nitrogen-doped carbon nanotube arrays can be used as promising support materials for ORR catalysts. The heteroatom doping strategy has been extended greatly through changing the doped atoms or introducing different atoms simultaneously in the past years. ${ }^{[21]}$

It should be noted that the carbon supports discussed above have their unique characteristics, which provides another possible way to design the support through compositing at least two supports together. As reported, the Pt particles loaded on porous carbon nanofiber (PCNF) and CB composite were used as cathode catalysts to fabricate MEA, which exhibited enhanced maximum power density (2 times in comparison with $\mathrm{Pt} / \mathrm{C}$ catalyst). ${ }^{[22]}$ It also has been reported that the reduced graphene oxide sheet that collaborated with CB can enhance the durability of catalyst through recapturing the dissolved $\mathrm{Pt}$ species. ${ }^{[23]}$ With respect to the $\mathrm{Pt} / \mathrm{C}$, the 100 -hour stability test of the bimetallic pyrolyzed NiCo-Zeolitic Imidazolate Framework (ZIF) supported fine $\mathrm{Pt} / \mathrm{Pt}$ alloy electrocatalyst showed no degradation under the MEA test conditions. ${ }^{[24]}$ Further researches reinforce that the superior performance is credited to the void 3D framework of $\mathrm{N}$-doped nanoporous carbon and the rich defect on the carbon support.

To address the issue of catalyst support corrosion, some alternative non-carbonaceous support materials also have been explored in the past decades. The titanium oxide and tin oxide have been proved more stable under high potential fuel cell environments, which are promising materials to prevent the agglomeration of the catalyst caused by carbon corrosion. ${ }^{[25]}$ In view of the different mechanism that generates high 
conductivity, the tin oxide and titanium oxide are used as the support materials of anode and cathode catalyst, respectively.

\section{lonomers}

\section{lonomer type}

As the binder in catalyst layers, ionomers play an essential role in supporting, binding the catalysts, and providing proton conductivity in the catalyst layer. For the choice of ionomers, mechanical strength, thermal stability, and proton conductivity are the basic considerations. Perfluorosulfonic acid (PFSA) ionomers, which are made of a hydrophobic perfluorinated backbone with sulfonated perfluoropolyether as side chains, have been selected as the preferable ionomer for PEMFCs. According to the differences in the length of side chains, PFSA ionomers are usually divided into two kinds: long-side-chain (LSC) ionomers and short-side-chain (SSC) ionomers.

LSC ionomers, especially Nafion, are most widely used in research and manufacture. However, LSC ionomers have some inherent weaknesses, such as relatively low operation temperature $\left(<90{ }^{\circ} \mathrm{C}\right)$, insufficient water-absorbing capacity, and poor proton conductivity under dry conditions. These disadvantages limit the development of PEMFCs in vehicles, which are desirable to operate at a higher temperature and lower humidity to achieve higher performance, better carbon monoxide tolerance, and more simplified cooling and humidification systems.

As the synthesis process of monomers is simplified and the cost is reduced, SSC ionomers have caught more attention in the last few years. SSC ionomers can overcome many of the defects of LSC ionomers. Due to shorter side chains (Figure 4), the ionomers possess higher crystallinity, higher thermal transition temperature, better water-absorbing capacity, and higher proton conductivity, which promise bright prospects in high-temperature proton exchange membrane fuel cells (HT-PEMFCs). ${ }^{[26]}$

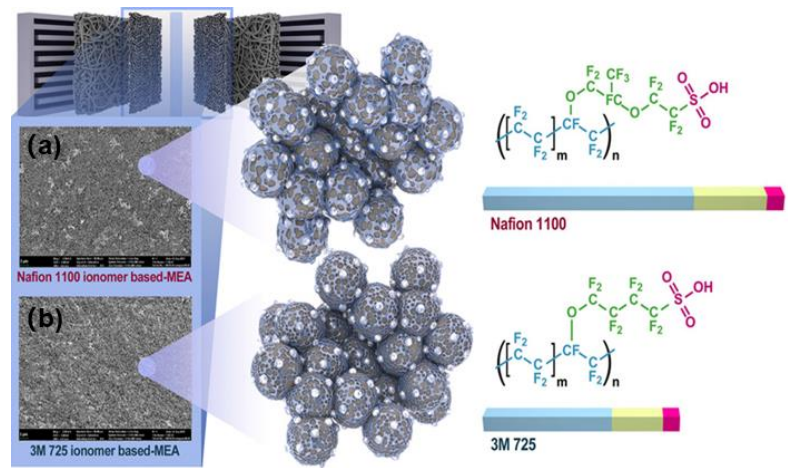

Figure 4 The molecular structure, the ionomer morphology in catalyst ink dispersions, and field emission scanning electron microscope (FESEM) surface images of the catalyst layer in the MEA: (a) Nafion 1100; (b) 3M 725 ionomers. ${ }^{[27]}$ Reprinted with permission from Ref. [27]. Copyright 2018 John Willey and Sons.

Meanwhile, many researchers have investigated the influence of the SSC ionomers on the performance and durability of low-temperature proton exchange membrane fuel cells (LT-PEMFCs) in comparison with the LSC PFSA ionomers. It has been commonly accepted that short side-chain ionomers are preferable to be the binders. ${ }^{[27-33]}$ It has been greatly agreed that the proton conductivity can be improved with the increase of relative humidity. ${ }^{[34]}$ Since the SSC ionomers usually have lower equivalent weight (EW) and better water absorption capability, their proton conductivities are higher than those of LSC ionomers, especially under low humidity conditions, resulting in a substantial performance improvement. ${ }^{[33]}$ On the other hand, SSC ionomers possess higher mobility and stronger intermolecular force, leading to increased phase separation and anisotropy. Consequently, SSC ionomers are expected to show a relatively weaker clustering trend and thus form smaller agglomerated structures (Figure 4). ${ }^{[27]}$ More homogeneous catalyst and ionomer agglomerates improve the platinum utilization, increase the proportion of micropores, and reduce the oxygen transportation resistance. ${ }^{[27,29]}$ Besides, some researches have revealed that MEA prepared with SSC ionomers possess higher durability than those prepared with LSC ionomers. ${ }^{[29]}$ However, compared with the LSC ionomers, the chemical stability of the SSC ionomers is required to be further improved.

In terms of a certain kind of ionomers, EW is a significant parameter that must be taken into consideration. EW has a great effect on the dispersion of catalysts and ionomers. Generally, lower EW means higher proton conductivity and better water-absorbing capability. Nevertheless, when the equivalent weight is reduced to a certain value, the ionomer thin films would swell or even dissolve, which causes the reduction of proton conductivity. Therefore, the choice of equivalent requires a trade-off.

\section{lonomer content}

The ratio of ionomer to carbon support $(\mathrm{I} / \mathrm{C})$ has been demonstrated as the main parameter that needs to be optimized due to its great impact on the performance and long-term durability of the PEMFCs. Inadequate ionomers are incapable to provide sufficient coverage to the catalysts and form a complete ionomer network. In this case, insufficient three-phase boundaries and high proton transport resistance result in a decrease in performance. When I/C ratio exceeds appropriate range, excess ionomers can lead to a significant increase in mass resistance. ${ }^{[35]}$

The mass resistance mainly includes local resistance and bulk resistance. Suzuki et al. ${ }^{[36]}$ developed a model of oxygen transport and considered that oxygen dissolution resistance on the interface of gas/ionomer is a significant part of local resistance, while the increase of thickness of ionomer film could attribute little to local resistance. Nevertheless, Wang et al. ${ }^{[37]}$ figured out that the increase of ionomer content may prolong the oxygen diffusion pathway, thus, it could produce extra resistance more than those caused by the increase of thickness of ionomer films (Figure 5a). Based on the models of local resistance, Doo et al. ${ }^{[38]}$ used molecular masking to selectively prevent the ionomers from deposition on the Pt surface, which reduced local resistance at the same ionomer content and thus improved the performance of the MEA.

(a)
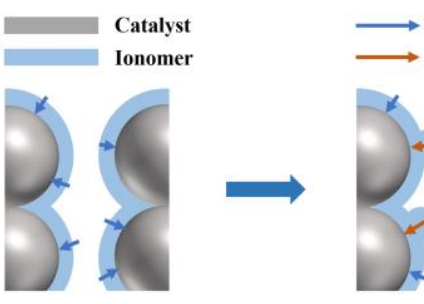
$\mathrm{O}_{2}$ transport path
Prelonged $\mathrm{O}_{2}$ transport path

(b)
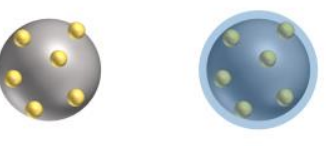

Low I/C

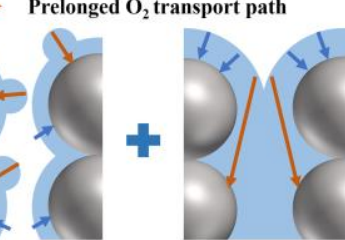

High I/C
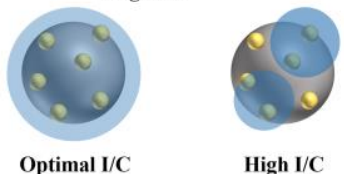

High I/C

Figure 5 Schematic of the structure evolution on the electrode interface with increasing content of the ionomer: (a) the transport path model; (b) the distribution model. 
In the thin catalyst layers with relatively low Pt loading, reactants could be transported to the side away from the GDL easily, which means local resistance plays a leading role. As the thickness of the catalyst layer increase with the Pt loading, bulk resistance shows high sensitivity to $\mathrm{I} / \mathrm{C}$ ratio and has a greater effect on performance. As the carbon network is formed by the combination of carbon aggregates, there are some macropores named secondary pores among the aggregates, which are the pathway for water and reactant gas. Also, there are some mesopores and micropores in the carbon aggregates, which are called primary pores. With the content of the ionomer increasing, the secondary pores will be gradually filled, and then will the primary pores. ${ }^{[3]}$ Because the pathway is blocked or narrowed by the ionomer, many of the reactants cannot cross the ionomer aggregates and reach the active sites. Since water cannot be discharged in time, the active sites would be immersed in water. The water flooding severely hinders the transport of reactants, causing a drop in performance.

Andersen et al. ${ }^{[39]}$ have proposed a novel potential mechanism to explain the effects of the ionomer content. They believed that as the ionomer content increases, the ionomers tended to form large clusters during the process of ink preparation and ink application, resulting in the inhomogeneous distribution of ionomers on the surface of catalysts (Figure 5b). Therefore, some Pt particles were not covered by ionomers. The decrease of the three-phase boundary causes the fall of performance as the ionomer content increases. However, the dispersity of ionomers is well affected by the solvents and mixing process. The actual reason for this phenomenon may be uneven ink dispersion. The hypothesis needs to be further verified by eliminating the influence of dispersion.

In addition to the remarkable impact on the performance, the ionomer content also has significant effects on the degradation of catalysts. Andersen et al. ${ }^{[39]}$ have proved that surface coverage of the ionomer on catalyst could effectively prevent platinum nanoparticles from degradation. When the ionomer was adequate to provide sufficient coverage, the long-term durability of MEAs could be well improved. What's more, in their research, the optimal performance and durability were achieved at the same ionomer content, suggesting that the most suitable ionomer coverage for performance and durability may be very close.

\section{Solvents}

Solvents, dispersion media of catalysts and ionomers, are generally a significant factor to control viscosity, dispersion, and stability of catalyst inks. Since the catalyst layer is fabricated by spraying or brushing with the ink, the ionomer distribution in the catalyst layer can be controlled by the solvent composition, which has direct effects on the ionomer aggregate size and ionomer/support interaction. Therefore, the adjustment of solvents is essential to optimize the catalyst layer.

As mentioned in the ionomer section, PFSA ionomers consist of a hydrophobic perfluorinated backbone and hydrophilic sulfonic acid groups on the side chains. In aqueous solvents like water, the hydrophobic backbone of PSFA ionomer tended to aggregate, while the sulfonic acid groups tended to be extended. On the contrary, in a nonaqueous solvent, such as dipropylene glycol (DPG), hydrophobic interaction with the solvent causes the extension of the backbone of PFSA ionomer. Molecular dynamics (MD) simulation suggests that in the water/DPG solvent system, the stronger affinity of the PFSA ionomer backbone for DPG solvent results in dispersion of ionomers at the nanoscale, while the increase in water content reduced the affinity, leading to the aggregation of backbones. ${ }^{[40]}$ Due to ionomer/solvents interaction, aggregates size distribu- tion varies in different solvents. Moreover, in mixing solvents, the aggregation of PFSA ionomer was determined by independent aggregation behaviors of backbone parts and sulfonic acid groups, which suggests that the size of aggregates can be tuned by changing the ratio of solvents. Generally, the increase in the aqueous solvent content leads to the enlargement of aggregates, which has been proved in water/DPG and water/propanol solvent systems. ${ }^{[40,41]}$

Doo et al. ${ }^{[40]}$ put forward an interesting model to explain the distribution of ionomers in the catalyst layers, which is based on the relative size of catalyst and ionomer aggregates in the ink. The model indicates that only when the sizes of catalyst and ionomer aggregates are comparable, a homogeneous ionomer network could be formed (Figure 6b). In this case, proton transfer and mass transfer can reach equilibrium to achieve optimized performance. Otherwise, sufficiently small ionomer aggregates may fill up the mesopores, which would hinder mass transfer (Figure 6a). Also, overlarge ionomer aggregates may form an incomplete ionomer network and block the macropores, which make up the main pathway of reactant gas and water, causing poor mass and proton transfer (Figure 6c).
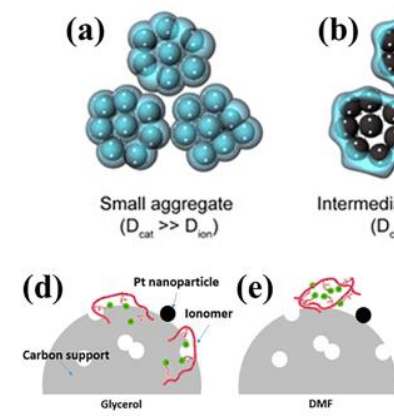
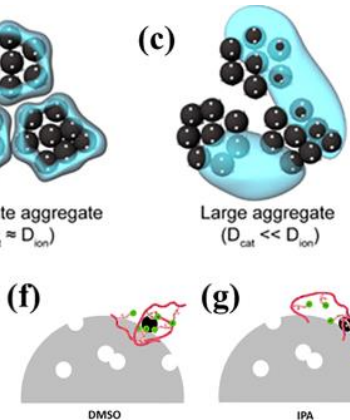

(g)

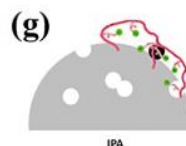

Figure 6 Models to illustrate the distribution of ionomers in the catalyst layer: (a), (b), (c) size effect of the ionomer aggregates; (d), (e), (f), (g) preferential coverage of the ionomer. ${ }^{[40,42]}$ Modified with permission from Ref. [40] and Ref. [42]. Copyright 2018 American Chemical Society.

The ionomer/support interaction is another significant factor that has a direct effect on the ionomer distribution. Due to different physical and chemical properties of solvents, ionomer/support interaction would also show obvious differences. Sharma et al. ${ }^{[42]}$ pointed out that solvents may affect the preference of ionomers in combination with catalyst nanoparticles or support. They used different solvents to prepare catalyst ink and developed different catalyst/ionomer interface structure. Based on the impedance measurement, the interface of catalysts and ionomers was interpreted as the ionomers have different preferential coverage of catalyst nanoparticles and support (Figures $6 \mathrm{~d}-6 \mathrm{~g}$ ).

Sharma et al. ${ }^{[43]}$ further observed ionomer/support interface directly by SEM and EDX. They dropped inks composed of different solvents on the gold plate. It was found that ionomer/support interface could be homogeneous, partially inhomogeneous, or completely inhomogeneous, which was affected by the interaction between ionomers and solvents. Since only a homogeneous structure can ensure both proton transfer and mass transfer, it is crucial to choose solvents that possess not only proper solubility but also sufficiently strong interaction.

\section{Conclusions and Perspectives}

This minireview summarizes recent advances on the component optimization for catalyst layers in PEMFCs, focusing on the support materials, ionomers, and solvents. In consideration of the superiority of carbon materials and its 
degradation mechanisms in fuel cells, the rational design of structure, surface modification, and heteroatom doping have been developed in previous studies as the strategies to hinder carbon corrosion. In terms of performance and durability, SSC ionomers are more suitable alternative options as the binder in the catalyst layers for PEMFCs. The choice of EW and the optimization of ionomer content are foundational but effective ways to further improve the performance. Suitable aggregate sizes and homogenous distribution of ionomers are largely dependent on physical and chemical properties of solvents.

However, there still exists great room for the development of component optimization. The intrinsic properties of support materials and ionomers require improvement. Furthermore, the interactions between catalysts and support materials also need to be further explored. Finally, the effect of solvents on the ionomer distribution needs to be further revealed.

\section{Acknowledgement}

The authors acknowledge financial support from the National Key R\&D Program of China (No. 2017YFB0406000), the National Natural Science Foundation of China (Nos. 21875137, 51521004, 51420105009), the Innovation Program of Shanghai Municipal Education Commission (Grant No. 2019-01-07-00-02-E00069), the 111 Project (Grant No. B16032), and the fund from Center of Hydrogen Science and Joint Research Center for Clean Energy Materials for financial supports.

\section{Author Contributions}

The following are the details of the contributions made by each of the authors for the manuscript: J. P. and W. Z. contributed equally to this work. J. W. oversaw the project. X. Z. and T. D. helped to revise the paper. W. S., P. T, and C. S. also helped to discuss the manuscript. All authors discussed the results and contributed to the manuscript.

Copyright $\odot 2021$ Jiaheng Peng, Wencong Zhang, Xuewei Zhang, Peng Tao, Chengyi Song, Wen Shang, Tao Deng, Jianbo Wu. This article is an open access article distributed under the terms and conditions of the Creative Commons Attribution (CC BY) license (http://creativecommons. org/licenses/by/4.0/). The use, distribution or reproduction in other forums is permitted, provided the original author(s) or licensor are credited and that the original publication in this journal is cited, in accordance with accepted academic practice. No use, distribution or reproduction is permitted which does not comply with these terms.

\section{References}

[1] Debe, M. K. Electrocatalyst approaches and challenges for automotive fuel cells. Nature 2012, 486, 43-51.

[2] Liu, M.; Zhao, Z.; Duan, X.; Huang, Y. Nanoscale Structure Design for High-Performance Pt-Based ORR Catalysts. Adv. Mater. 2019, 31, 1802234.

[3] $\mathrm{Wu}$, J.; Yang, H. Platinum-based oxygen reduction electrocatalysts. Acc. Chem. Res. 2013, 46, 1848-1857.

[4] Stephens, I. E. L.; Rossmeisl, J.; Chorkendorff, I. Toward sustainable fuel cells. Science 2016, 354, 1378-1379.

[5] Egetenmeyer, A.; Radev, I.; Durneata, D.; Baumgärtner, M.; Peinecke, V.; Natter, H.; Hempelmann, R. Pulse electrodeposited cathode catalyst layers for PEM fuel cells. Int. J. Hydrogen Energy 2017, 42, 13649-13660.

[6] Ostroverkh, A.; Johánek, V.; Dubau, M.; Kúš, P.; Khalakhan, I.; Šmíd, B.; Fiala, R.; Václavů, M.; Ostroverkh, Y.; Matolín, V. Optimization of ionomer-free ultra-low loading $\mathrm{Pt}$ catalyst for anode/cathode of PEMFC via magnetron sputtering. Int. J. Hydrogen Energy 2019, 44, 19344-19356.
[7] Saha, M. S.; Gullá, A. F.; Allen, R. J.; Mukerjee, S. High performance polymer electrolyte fuel cells with ultra-low Pt loading electrodes prepared by dual ion-beam assisted deposition. Electrochim. Acta 2006, 51, 4680-4692.

[8] Sasaki, K.; Naohara, H.; Cai, Y.; Choi, Y. M.; Liu, P.; Vukmirovic, M. B.; Wang, J. X.; Adzic, R. R. Core-protected platinum monolayer shell high-stability electrocatalysts for fuel-cell cathodes. Angew. Chem. 2010, 122, 8784-8789.

[9] Lefèvre, M.; Proietti, E.; Jaouen, F.; Dodelet, J. P. Iron-based catalysts with improved oxygen reduction activity in polymer electrolyte fuel cells. Science 2009, 324, 71-74.

[10] Wu, J.; Zhang, J.; Peng, Z.; Yang, S.; Wagner, F. T.; Yang, H. Truncated octahedral $\mathrm{Pt}_{3} \mathrm{Ni}$ oxygen reduction reaction electrocatalysts. J. Am. Chem. Soc. 2010, 132, 4984-4985.

[11] Chen, C.; Kang, Y.; Huo, Z.; Zhu, Z.; Huang, W.; Xin, H. L.; Snyder, J. D.; Li, D.; Herron, J. A.; Mavrikakis, M. Highly crystalline multimetallic nanoframes with three-dimensional electrocatalytic surfaces. Science 2014, 343, 1339-1343.

[12] Banham, D.; Ye, S. Current status and future development of catalyst materials and catalyst layers for proton exchange membrane fuel cells: An industrial perspective. ACS Energy Lett. 2017, 2, 629-638.

[13] Zheng, J. S.; Wang, M. X.; Zhang, X. S.; Wu, Y. X.; Li, P.; Zhou, X. G.; Yuan, W. K. Platinum/carbon nanofiber nanocomposite synthesized by electrophoretic deposition as electrocatalyst for oxygen reduction. J. Power Sources 2008, 175, 211-216.

[14] Zheng, J. S.; Wang, X. Z.; Fu, R.; Yang, D. J.; Li, P.; Lv, H.; Ma, J. X. Microstructure effect of carbon nanofibers on Pt/CNFs electrocatalyst for oxygen reduction. Int. J. Hydrogen Energy 2012 37, 4639-4647.

[15] Jafri, R. I.; Rajalakshmi, N.; Ramaprabhu, S. Nitrogen doped graphene nanoplatelets as catalyst support for oxygen reduction reaction in proton exchange membrane fuel cell. J. Mater. Chem. 2010, 20, 7114-7117.

[16] Viva, F. A.; Bruno, M. M.; Franceschini, E. A.; Thomas, Y. R.; Sanchez, G. R.; Solorza-Feria, O.; Corti, H. R. Mesoporous carbon as Pt support for PEM fuel cell. Int. J. Hydrogen Energy 2014, 39, 8821-8826.

[17] King, J. S.; Wittstock, A.; Biener, J.; Kucheyev, S. O.; Wang, Y. M.; Baumann, T. F.; Giri, S. K.; Hamza, A. V.; Baeumer, M.; Bent, S. F. Ultralow loading $P t$ nanocatalysts prepared by atomic layer deposition on carbon aerogels. Nano Lett. 2008, 8, 2405-2409.

[18] Gerber, I. C.; Serp, P. A Theory/Experience Description of Support Effects in Carbon-Supported Catalysts. Chem. Rev. 2019, 120, $1250-1349$.

[19] Lu, X.; Yim, W. L.; Suryanto, B. H. R.; Zhao, C. Electrocatalytic oxygen evolution at surface-oxidized multiwall carbon nanotubes. J. Am. Chem. Soc. 2015, 137, 2901-2907.

[20] Gong, K.; Du, F.; Xia, Z.; Durstock, M.; Dai, L. Nitrogen-doped carbon nanotube arrays with high electrocatalytic activity for oxygen reduction. Science 2009, 323, 760-764.

[21] Gao, K.; Wang, B.; Tao, L.; Cunning, B. V.; Zhang, Z.; Wang, S.; Ruoff, R. S.; Qu, L. Efficient Metal-Free Electrocatalysts from $N$-Doped Carbon Nanomaterials: Mono-Doping and Co-Doping Adv. Mater. 2019, 31, 1805121.

[22] Mao, L.; Shan, C.; Fu, K.; Jin, J.; Yang, S.; Li, G. Porous Carbon Nanofiber/Carbon Black Composite as Promising Support for Platinum Catalyst to Enhance Oxygen Reduction Reaction in PEMFC. ChemistrySelect 2019, 4, 10555-10560.

[23] Li, Y.; Li, Y.; Zhu, E.; McLouth, T.; Chiu, C. Y.; Huang, X.; Huang, Y. Stabilization of high-performance oxygen reduction reaction pt electrocatalyst supported on reduced graphene oxide/carbon black composite. J. Am. Chem. Soc. 2012, 134, 12326-12329.

[24] Hanifa, S.; Shib, X.; Iqbala, N.; Noorc, T.; Anwar, R.; Kannanb, A. M. ZIF derived PtNiCo/NC cathode catalyst for proton exchange membrane fuel cell. Appl. Catal., B 2019, 258, 117947.

[25] Wang, Y. J.; P.Wilkinson, D.; Zhang, J. Noncarbon support 
materials for polymer electrolyte membrane fuel cell electrocatalysts. Chem. Rev. 2011, 111, 7625-7651.

[26] Stassi, A.; Gatto, I.; Passalacqua, E.; Antonucci, V.; Arico, A.; Merlo, L.; Oldani, C.; Pagano, E. Performance comparison of long and short-side chain perfluorosulfonic membranes for high temperature polymer electrolyte membrane fuel cell operation. J. Power Sources 2011, 196, 8925-8930.

[27] Kim, O. H.; Oh, S. H.; Ahn, C. Y.; Kim, S.; Kim, J. K.; Kim, J.; Yang, S.; Choi, M.; Cho, Y. H.; Sung, Y. E. Enhanced Performance of lonomer Binder with Shorter Side-Chains, Higher Dispersibility, and Lower Equivalent Weight. Fuel Cells 2018, 18, 711-722.

[28] Balogun, E. O.; Hussain, N.; Chamier, J.; Barendse, P. Performance and durability studies of perfluorosulfonic acid ionomers as binders in PEMFC catalyst layers using Electrochemical Impedance Spectroscopy. Int. J. Hydrogen Energy 2019, 44, 32219-32230.

[29] Shahgaldi, S.; Alaefour, I.; Li, X. The impact of short side chain ionomer on polymer electrolyte membrane fuel cell performance and durability. Appl. Energy 2018, 217, 295-302.

[30] Shahgaldi, S.; Alaefour, I.; Zhao, J.; Li, X. Impact of ionomer in the catalyst layers on proton exchange membrane fuel cell performance under different reactant flows and pressures. Fuel 2018, 227, 35-41.

[31] Talukdar, K.; Gazdzicki, P.; Friedrich, K. A. Comparative investigation into the performance and durability of long and short side chain ionomers in Polymer Electrolyte Membrane Fuel Cells. J. Power Sources 2019, 439, 227078.

[32] Peron, J.; Edwards, D.; Haldane, M.; Luo, X.; Zhang, Y.; Holdcroft, S.; Shi, Z. Fuel cell catalyst layers containing short-side-chain perfluorosulfonic acid ionomers. J. Power Sources 2011, 196, 179-181.

[33] Garsany, Y.; Atkinson III, R. W.; Sassin, M. B.; Hjelm, R. M.; Gould, B. D.; Swider-Lyons, K. E. Improving PEMFC performance using short-side-chain low-equivalent-weight PFSA ionomer in the cathode catalyst layer. J. Electrochem. Soc. 2018, 165, F381.

[34] Liu, Y.; Murphy, M. W.; Baker, D. R.; Gu, W.; Ji, C.; Jorne, J.; Gasteiger, H. A. Proton conduction and oxygen reduction kinetics in PEM fuel cell cathodes: effects of ionomer-to-carbon ratio and relative humidity. J. Electrochem. Soc. 2009, 156, B970.

[35] Chen, L.; Zhang, R.; Kang, Q.; Tao, W.-Q. Pore-scale study of pore-ionomer interfacial reactive transport processes in proton exchange membrane fuel cell catalyst layer. Chem. Eng. J. 2020, 391,123590 .

[36] Suzuki, T.; Kudo, K.; Morimoto, Y. Model for investigation of oxygen transport limitation in a polymer electrolyte fuel cell. J. Power Sources 2013, 222, 379-389.

[37] Wang, C.; Cheng, X.; Yan, X.; Shen, S.; Ke, C.; Wei, G.; Zhang, J. Respective Influence of Ionomer Content on Local and Bulk Oxygen Transport Resistance in the Catalyst Layer of PEMFCs with Low Pt Loading. J. Electrochem. Soc. 2019, 166, F239.

[38] Doo, G.; Yuk, S.; Lee, J. H.; Choi, S.; Lee, D.-H.; Lee, D. W.; Hyun, J.; Kwon, S. H.; Lee, S. G.; Kim, H.-T. Nano-scale control of the ionomer distribution by molecular masking of the Pt surface in PEMFCs. J. Mater. Chem. A 2020, 8, 13004-13013.

[39] Andersen, S. M.; Grahl-Madsen, L. Interface contribution to the electrode performance of proton exchange membrane fuel cells-Impact of the ionomer. Int. J. Hydrogen Energy 2016, 41, 1892-1901.

[40] Doo, G.; Lee, J. H.; Yuk, S.; Choi, S.; Lee, D. H.; Lee, D. W.; Kim, H. G.; Kwon, S. H.; Lee, S. G.; Kim, H. T. Tuning the ionomer distribution in the fuel cell catalyst layer with scaling the ionomer aggregate size in dispersion. ACS Appl. Mater. Interfaces 2018, 10, 17835-17841.

[41] Orfanidi, A.; Rheinländer, P. J.; Schulte, N.; Gasteiger, H. A. Ink Solvent Dependence of the Ionomer Distribution in the Catalyst Layer of a PEMFC. J. Electrochem. Soc. 2018, 165, F1254.

[42] Sharma, R.; Andersen, S. M. Zoom in catalyst/ionomer interface in polymer electrolyte membrane fuel cell electrodes: Impact of catalyst/ionomer dispersion media/solvent. ACS Appl. Mater. Interfaces 2018, 10, 38125-38133.

[43] Sharma, R.; Grahl-Madsen, L.; Andersen, S. M. Influence of dispersion media on Nafion $\circledast$ ionomer distribution in proton exchange membrane fuel cell catalyst carbon support. Mater. Chem. Phys. 2019, 226, 66-72.

Received October 3, 2020 Accepted November 21, 2020 